\title{
Retrospective outcomes analysis of 99 consecutive uniportal awake lung biopsies: a real standard of care?
}

\author{
Roberto Cherchi ${ }^{1}$, Giulia Grimaldi ${ }^{1}$, Matteo Pinna-Susnik ${ }^{1}$, Laura Riva ${ }^{1}$, Sabrina Sarais ${ }^{1}$, \\ Massimiliano Santoru ${ }^{1}$, Roberto Perra ${ }^{2}$, Roberto Allieri ${ }^{3}$, Giuseppe S. Porcu ${ }^{4}$, Sonia Nemolato ${ }^{4}$, \\ Antonella Mameli ${ }^{5}$, Federica Loi ${ }^{6}$, Paolo A. Ferrari ${ }^{1}$
}

${ }^{1}$ Division of Thoracic Surgery, “A. Businco” Oncology Hospital, Azienda Ospedaliera Brotzu, Cagliari, Italy; ${ }^{2}$ Pulmonology Unit and Respiratory Physiopathology Laboratory, "R. Binaghi” and "SS. Trinità" Hospitals, ATS Sardegna, Cagliari, Italy; ${ }^{3}$ Radiology Service, "R. Binaghi” Hospital, ATS Sardegna, Cagliari, Italy; ${ }^{4}$ Histopathology Department, “A. Businco” Oncology Hospital, Azienda Ospedaliera Brotzu, Cagliari, Italy; ${ }^{5}$ Internal Medicine and Haemocoagulopathies Division, AOU of Monserrato, Monserrato, Italy; ${ }^{6}$ Sardinian Epidemiological Observatory, IZS of Sardinia, Cagliari, Italy

Contributions: (I) Conception and design: R Cherchi, PA Ferrari; (II) Administrative support: R Cherchi, PA Ferrari; (III) Provision of study materials or patients: G Grimaldi, M Pinna-Susnik, L Riva, S Sarais, M Santoru, R Perra, R Allieri, GS Porcu, S Nemolato, A Mameli; (IV) Collection and assembly of data: F Loi, M Pinna-Susnik; (V) Data analysis and interpretation: F Loi, PA Ferrari; (VI) Manuscript writing: All authors; (VII) Final approval manuscript: All authors.

Correspondence to: Paolo A. Ferrari. Division of Thoracic Surgery, “A. Businco” Cancer Center, Azienda Ospedaliera Brotzu, Via Jenner 1, 09121 Cagliari, Italy. Email: paoloalb.ferrari@gmail.com.

Background: Surgical lung biopsy for interstitial lung disease (ILD) is traditionally performed through video-assisted thoracic surgery (VATS) and general anesthesia (GA). The mortality and morbidity rates associated with this procedure are not negligible, especially in patients with significant risk factors and respiratory impairment. Based on these considerations, our center evaluated a safe non-intubated VATS approach for lung biopsy performed in ILD subjects.

Methods: Ninety-nine patients affected by undetermined ILD were enrolled in a retrospective cohort study. In all instances, lung biopsies were performed using a non-intubated VATS technique, in spontaneously breathing patients, with or without intercostal nerve blockage. The primary end-point was the diagnostic yield, while surgical and global operating room times, post-operative length of stay (pLOS), numeric pain rating scale (NPRS) after surgery and early mortality were considered as secondary outcomes.

Results: All the procedures were carried out without conversion to GA. The pathological diagnosis was achieved in 97 patients with a diagnostic yield of $98 \%$. The mean operating room length-of-stay and operating time were 73.7 and $42.5 \mathrm{~min}$, respectively. Mean pLOS was 1.3 days with a low readmissions rate (3\%). No mortality in the first 30 days due to acute exacerbation of ILD occurred. Both analgesia methods resulted in optimal feasibility with a mean NPRS score of 1.13 .

Conclusions: In undetermined ILD patients, surgical lung biopsy with a non-intubated VATS approach and spontaneous ventilation anesthesia appears to be both a practical and safe technique with an excellent diagnostic yield and high level of patient satisfaction.

Keywords: Awake surgery; interstitial lung disease (ILD); non-intubated video-assisted thoracic surgery (NIVATS); surgical lung biopsy (SLB); tubeless anesthesia

Submitted Apr 02, 2020. Accepted for publication Aug 03, 2020.

doi: $10.21037 /$ jtd-20-1551

View this article at: http://dx.doi.org/10.21037/jtd-20-1551 


\section{Introduction}

As reported by international guidelines, in the correct clinical setting with all possible causes of interstitial lung disease (ILD) ruled out, the diagnosis of idiopathic pulmonary fibrosis (IPF) is adequate when a clear, usual interstitial pneumonia (UIP) pattern is identified on chest high resolution computed tomography (HRCT). Conversely, a surgical lung biopsy (SLB) should be considered in patients with a HRCT pattern of probable UIP, indeterminate for UIP or alternative diagnosis (1). Relevant information available is then analyzed in a multidisciplinary discussion (MDD) among clinicians, radiologists and pathologists, all being experts in the field of ILD, in order to increase the accuracy of the diagnosis (2) and management (3). For these reasons MDD is strongly suggested in the evaluation of ILD, when clinical, radiological, and/or histological data is not univocal $(1,4)$.

One challenge with this diagnostic approach is that many patients with a HRCT pattern of probable or indeterminate with UIP do not undergo a SLB. The decision whether to perform a surgical biopsy depends upon the perceived benefit derived from histopathologic examination, balanced against the perceived mortality and morbidity of the procedure (5), related to age, disease severity or comorbidities (6). Every clinician and thoracic surgeon routinely needs to evaluate the benefits and risks of a lung biopsy in patients with ILD, and while the benefit of a secure and tentatively early diagnosis is now well established, the literature evaluating the risk associated with a SLB is scarce and often disagrees. Furthermore, international guidelines as well as new targeted therapies for IPF, have highlighted the need for accurate diagnosis of ILD, which may lead an increase in SLB procedures (7). Video-assisted thoracoscopic surgery (VATS) is the preferred approach to lung biopsy for patients who can tolerate single-lung ventilation, rather than open thoracotomy $(1,8,9)$. Pooling studies (unweighted) indicated that SLB obtained an adequate sample in all patients $(100 \%$; 95\% CI, 99-100\%), although the experts acknowledged that this is not always the case in clinical practice; the diagnostic yield of SLB was high $(88.2 \%$; 95\% CI, 86.9$89.4 \%$ ), with a minority of unclassifiable histopathological reports (11.8\%; 95\% CI, 10.6-13.1\%) (1).

The caution needed when referring ILD patients for surgical biopsy derives from a growing concern for the rate of mortality, ranging between $4.2 \%$ and $17.5 \%$ depending on the stratification of risk factors (10), considering the acute exacerbation of ILD after biopsy as being one of the major causes of mortality. Thus, in recent years, nonintubated VATS (NIVATS) procedures have been carried out in order to avoid the operating and anesthesiological risks of general anesthesia (GA), preserving an acceptable diagnostic and curative outcome, through the avoidance of muscle relaxants and lung injuries from positive pressure ventilation $(11,12)$.

Based on these considerations our Center proposed a standardized NIVATS approach for lung biopsy in ILD patients, performed under local anesthesia and sedation. The aim of the study was to evaluate the suitability of this technique, based on its capacity to perform accurate diagnosis on adequate biopsy samples, as well as to define relevant advantages and disadvantages.

Preliminary considerations have been formulated regarding the possibility of applying this procedure as a valid alternative to intubated VATS lung biopsy for patients with newly detected ILD of apparently unknown causes.

We present the following article in accordance with the STROBE reporting checklist (available at http://dx.doi. org/10.21037/jtd-20-1551).

\section{Methods}

\section{Study population}

The study was approved by the Institutional Review Board (Reference Ethics Committee No. PG/2017/16770) and conducted in accordance with the Declaration of Helsinki (as revised in 2013). Informed written consent was obtained from all the patients. We retrospectively analyzed prospectively collected data of ILD subjects undergoing NIVATS lung biopsy at our Institute between April 2015 and August 2019.

An overall sample of 99 participants affected by undetermined ILD were recruited in order to detect a specific diagnosis in at least $95 \%$ patients undergoing NIVATS lung biopsy, starting from the proportion of $88 \%$ success rate of SLB described in the last review (1) with a power of $80 \%$ ( $\alpha=0.05$, two-sided), and considering a $5 \%$ of drop-out procedures.

The eligibility criteria included clinical findings of undetermined ILD, presented by pulmonologists in MDD (with thoracic surgeons, pathologists and dedicated radiologists) and deemed to require SLB for conclusive diagnosis. A standard pre-operative surgical work-up provided HRCT, pulmonary function tests (PFTs) and 


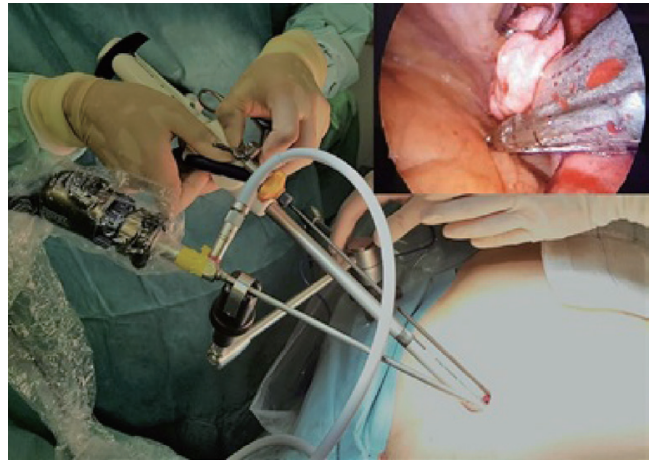

Figure 1 Uniportal NIVATS procedure for lung biopsy. Division of Thoracic Surgery, “A. Businco" Oncology Hospital, Cagliari (IT). NIVATS, non-intubated video-assisted thoracic surgery.

respiratory endoscopy evaluation with bronchial samples collection in most of the cases. Patients were excluded from this technique if any of the following were present: age $<18$ years, hemodynamic instability, patient already intubated and ventilated, anticipated need for extensive decortication. Patients with good PFTs results, a safe American Society of Anesthesiologist (ASA) score and a low Charlson comorbidity score (CHs) were treated as well as compromised respiratory patients with higher perioperative risk.

\section{Surgical procedure and management of anesthesia}

In order to perform the surgery, patients were positioned in lateral decubitus and moderate anti-Trendelenburg using soft pillows to assure comfort and stability. Standard vital and physiologic monitoring included venous catheterization, electrocardiogram, heart rate, pulse oximetry, non-invasive blood pressure and body temperature. Sterile drapes were placed on the patients leaving their face free in order to facilitate spontaneous breathing.

The operations started with the creation of surgical access after enough skin and subcutaneous tissue local infiltration with lidocaine $2 \%$. In our series, patients have been treated with chest wall local anesthesia and percutaneous intercostal nerve block (IB) with chirocaine $5 \%(\mathrm{n}=42)$ made by surgeon under thoracoscopic vision immediately after surgical access, while others received only chest wall local anesthesia $(n=58)$. Each patient was allocated a group according to patient consent and the preference of each surgeon. Pharmacological management with midazolam $(0.10-0.15 \mathrm{mg} / \mathrm{kg})$ was the standard intra- operative treatment for all patients, as well as $\mathrm{O}_{2}$ supply through nasal cannula or face mask. In order to prevent any possible cause of discomfort for the patient and to improve their compliance during the operation, conscious sedation with a total intra-venous anesthesia (TIVA) was preferentially offered and administered to reach or maintain a target Ramsey score 2-3 (13). A laryngeal mask airway and bronchial blocker were prepared in case of conversion to GA.

The lung biopsy was performed through the creation of pneumothorax by uniportal access at $5^{\text {th }}$ costal space (up to a maximum length of 3 centimeters) without ribs or soft tissue spreading, for 30-degree-5 millimeters angled camera and standard thoracoscopic instrumentation (Figure 1). The same surgical access was used for the retrieval of the specimen in all the patients, without spreading or enlargement. Intra-operative vagal nerve blockage or intrapleural local anesthetic nebulization was not required because only simple wedge resections were performed. The biopsy of lung region chosen to be the most suitable for diagnosis by radiologists and pulmonologists preoperatively was obtained under macroscopic visual validation. A single chest tube (CT) 19, 21 or 28 French (according to the preference of the surgeon) was placed through the uniportal chest access, under thoracoscopic vision after careful controls of hemostasis and aerostasis. Likewise, the reexpansion of the lung was confirmed and achieved by asking the patient to breathe deeply and cough effectively.

\section{Post-operative management and follow-up}

A routine evaluation via chest $\mathrm{X}$-ray (CXR) was requested within $4 \mathrm{~h}$ of the completion of the surgical procedures. The patient was declared dischargeable after removal of the CT, which was performed in the absence of an air-leak, checked with the CT under suction, with $<200 \mathrm{~mL} / 24 \mathrm{~h}$ of serous output. Patient feedback with regards the procedure was evaluated using a numeric pain rate scale (NPRS) report, obtained every $6 \mathrm{~h}$ from the time of readmission to the ward until discharge. The standard surgical followup for each patient was of a double clinical check and CXR evaluation up to 30 days post-discharge.

\section{Outcomes and statistical analysis}

Data quality was assessed in terms of accuracy, completeness, and missing information. Descriptive analyses were 
performed to evaluate the baseline distribution of each variable, for all patients undergoing NIVATS lung biopsy procedure. Most variables collected were categorical variables and were described by frequencies and percentage. Based on variable distribution, quantitative variables were expressed as mean values, standard deviations (SD), median, and interquartile range (IQR), as appropriate.

Any unexpected clinical event surpassing the standard clinical pathway was intended to be recorded as complication according to the Clavien-Dindo classification (14). The surgical operating room (OR) time was calculated as being that comprised between the initial skin incision and the completion of its closure, while the global OR time was calculated as the time between the beginning and the end of the anesthesia management. Finally, early mortality was defined as mortality within the first 30 days after surgery.

Based on the aim of the study, the primary endpoint of the analysis was the achievement of a successful final diagnosis to test the diagnostic yield of the applied technique. In addition, secondary outcomes as surgical OR time, global OR time, post-operative length of stay (pLOS), NPRS and early mortality was also considered. Their possible correlation with baseline characteristics such as body mass index (BMI), forced expiratory volume in 1 st second percentage (FEV1\%), forced vital capacity percentage (FVC\%) and the enrolment of subsequent patients were evaluated starting from standard clinical assumptions.

Considering the diverse managements in locoregional analgesia as a key point for the perception of pain in the patient, two different groups defining this factor were created. Post-operative outcomes such as surgical and global OR time, pLOS, NPRS and the demand of post-operative rescue analgesia were evaluated between the groups.

Possible differences in post-operative outcomes between patients treated with CT 19-21 French (designated small CT) or CT 28 French (designated large CT) were detected. Post-operative outcomes such as pLOS, NPRS and postoperative complications were evaluated between groups.

Post-operative outcomes have been tested based on different variables. The Shapiro-Wilk normality test was used to assess normal distribution of numeric variables. The unpaired Student's $t$-test was used for comparison of numeric variables with normal distribution and the MannWhitney test for those with non-normal distribution. The chi-square or Fisher's exact tests were used for categorical variables, as appropriate.

In the main analysis, the linear or nonlinear nature of the relationship between each of the continuous independent variables was first assessed graphically and, when a linear relationship was assumed, its strength was initially evaluated trough Pearson's parametric correlation coefficient. Linear regression models were fitted to quantify the effect of one or more variables on different outcomes for which a correlation was observed. Most relevant results have been reported in this study.

All statistical tests were two-tailed, with a significance level of 0.05. Bonferroni correction was applied for the multiple comparison tests. Stata ${ }^{\mathrm{TM}} 13.0$ statistical software (Stata Corp., College Station, TX, USA) was used for the analysis.

\section{Results}

\section{Descriptive analysis}

NIVATS lung biopsy procedures were successfully completed in all 99 patients eligible for the analysis and the 30-day post-surgery follow-up data was collected in all cases. Demographic variables, baseline characteristics and post-operative outcomes are summarized in descriptive statistic (Table 1) and the most representative are shown in Figure 2.

An adequate sample was successfully obtained in 97 out of the 99 patients (98\%) for histopathological diagnosis: UIP pattern was described in $60(61 \%)$ exams, probable UIP in $1(1 \%)$, indeterminate for UIP in $9(9 \%)$ and an alternative diagnosis was reported in $27(27 \%)$ of the collected resections. Only two samples $(2 \%)$ were considered to be inadequate/insufficient for diagnosis (Table 1).

The percentage of intra-operative peripheral oxygenation remained satisfactory through the operations with average of $97.2(\mathrm{SD}=2.2)$ and a mean of intra-operative $\mathrm{O}_{2} / \mathrm{L} / \mathrm{min}$ supply of $3.6(\mathrm{SD}=1.5)$. According to TIVA intra-operative management (Table 2), the surgery was completed without conversion to GA in all cases, even in patients with light to moderate pleural adhesions.

Overall, intra-operative complications occurred in 4 subjects (4\%) without the necessity of discontinuing the procedure: two patient showed limited respiratory impairment, one had minor bleeding and one registered psychomotor agitation. Furthermore, one procedure was aborted prior to sedation due to the patient severe psychomotor agitation and has not been included in this study; in this case after adequate psychological preparation, 
Table 1 Baseline characteristics and post-operative outcomes of patients who underwent NIVATS uniportal lung biopsy for undetermined ILD

\begin{tabular}{|c|c|}
\hline Variable & ILD patients $(n=99)$ \\
\hline \multicolumn{2}{|l|}{ Sex, $n[\%]$} \\
\hline Male & $73[74]$ \\
\hline Female & $26[26]$ \\
\hline Age (years), mean [SD] & $66[10]$ \\
\hline Ex-smokers & $61[62]$ \\
\hline No-smokers & $30[30]$ \\
\hline Body mass index (score), mean (SD) & $27.0(4.7)$ \\
\hline Forced expiratory volume in 1st second percentage (FEV1\%), mean (SD) & $86.3(23.1)$ \\
\hline Diffusing capacity for carbon monoxide percentage (DLCO\%), mean (SD) & $59.6(18.3)$ \\
\hline \multicolumn{2}{|l|}{ Post-operative complications, $\mathrm{n}$ [\%] } \\
\hline Yes & 9 [9] \\
\hline Not & $90[91]$ \\
\hline \multicolumn{2}{|l|}{ Decubitus, n [\%] } \\
\hline Left side & $48[48]$ \\
\hline Right side & $43[43]$ \\
\hline Left lower lobe (LLL) & $16[16]$ \\
\hline Right upper lobe (RUL) & $11[11]$ \\
\hline Middle lobe (ML) & $4[4]$ \\
\hline LUL + LLL & $5[6]$ \\
\hline$R L L+M L$ & $2[2]$ \\
\hline$R U L+R L L$ & $6[6]$ \\
\hline Number of lung biopsies, mean (SD) & $1.2(0.4)$ \\
\hline Average specimen length (centimeters), mean (SD) & $3.4(1.4)$ \\
\hline \multicolumn{2}{|l|}{ Feasibility, n [\%] } \\
\hline Easy & $53[54]$ \\
\hline Feasible (e.g., only one biopsy) & $33[33]$ \\
\hline Difficult (e.g., deeper sedation required) & $13[13]$ \\
\hline
\end{tabular}

Table 1 (continued) 
Table 1 (continued)

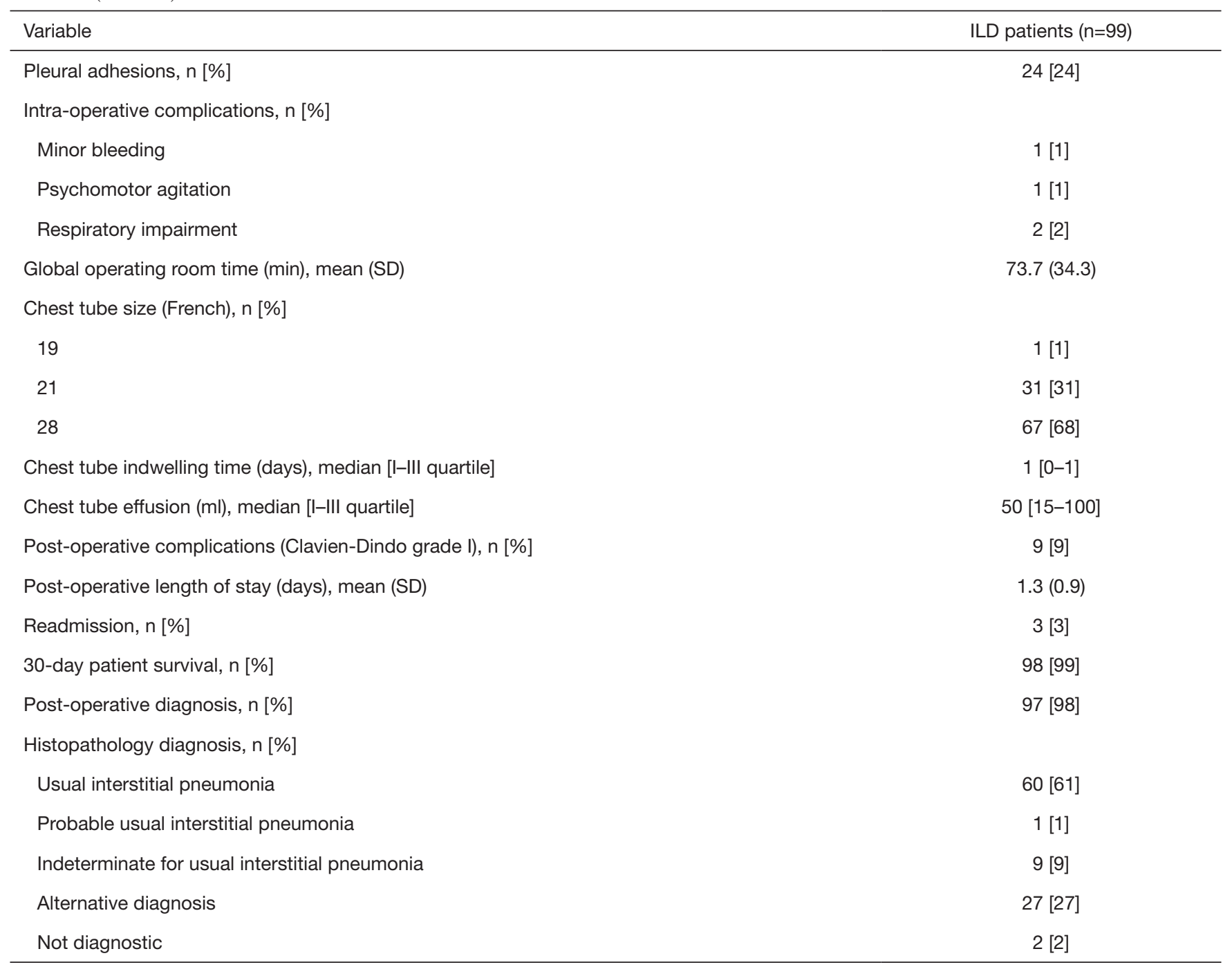

NIVATS, non-intubated video-assisted thoracic surgery; ILD, interstitial lung disease.

the procedure was successful performed 10 days later without intra-operative complications.

Analyzing the patients' post-operative feedback, the average NPRS was 1.13 (SD =0.82); 57 (58\%) patients requested analgesic supply during their post-operative hospital stay such as paracetamol and non-steroidal anti-inflammatory drugs (NSAID). Morphine was not administered to any patients (Table 2).

The average pLOS was $1.3(\mathrm{SD}=0.9)$ with $3(3 \%)$ of patients discharged the day of surgery. No in-hospital deaths were recorded in our series. According to the Clavien-Dindo classification (14) only nine patients had minor post-operative complications (Grade I), such as fever, pleural residual space and incomplete vasovagal reflex. As reported by clinical follow-up, one patient died due to encephalopathy secondary to acute liver insufficiency within 30 days after discharge, and two other patients died within 90 days for cardiovascular stroke. During the same followup observational time, only 3 patients (3\%) were readmitted for CXR manifestation of moderate pleural effusion that needed a medical thoracentesis, without further complications (Table 1).

\section{Secondary outcomes}

The progressive and subsequent number attributed to 

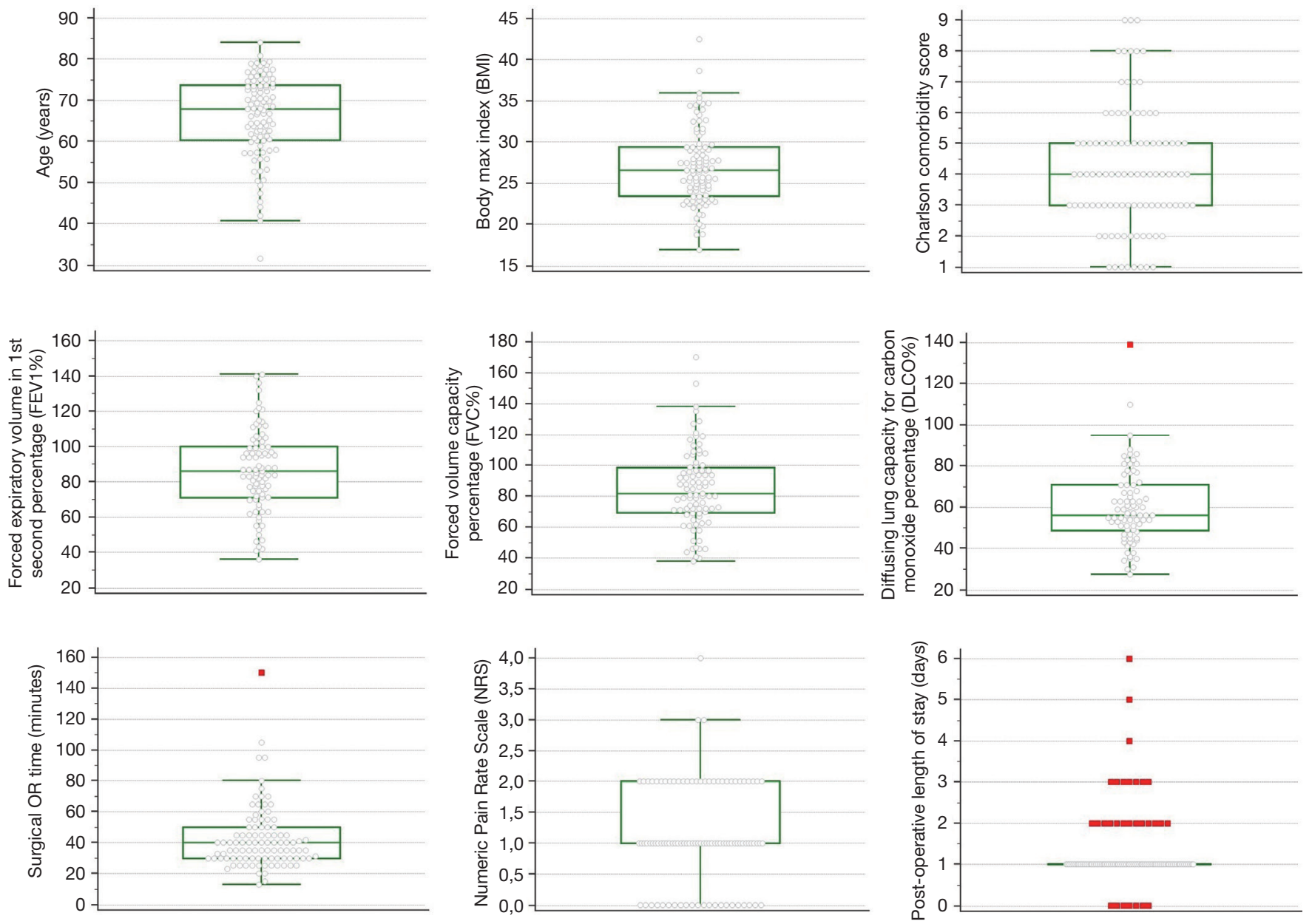

Figure 2 Main baseline characteristics and post-operative outcomes of patients who underwent NIVATS uniportal lung biopsy. NIVATS, non-intubated video-assisted thoracic surgery.

each patient upon admission (ID) was evaluated as a factor in helping to increase the expertise of the surgical team, supposing an inverse correlation with the surgical OR time. This hypothesis was confirmed by Pearson's correlation coefficients (Table S1) that demonstrated a statistically significant $(\mathrm{P}$ values $<0.05)$ moderate correlation between patient number and surgical OR time (Pearson's correlation coefficient $=-0.3033$ ).

Furthermore, the surgical OR time showed statistically significance negative moderate correlation with the preoperative FEV1\% ( $\mathrm{P}=0.0372)$ and $\mathrm{FVC} \%(\mathrm{P}=0.0444)$. Interestingly, the BMI was moderately positively correlated with surgical OR time and global OR time $(\mathrm{P}=0.0029$ and $\mathrm{P}=0.0321$ respectively). The difference in surgical $\mathrm{OR}$ time between patients with adhesions (mean $=52.5 \mathrm{~min}, \mathrm{SD}$ $=28.3 \mathrm{~min}$ ) and without (mean $=39.3 \mathrm{~min}, \mathrm{SD}=15.7 \mathrm{~min}$ ) was found to be statistically significant $(\mathrm{P}=0.005)$.

Predictably, pLOS showed statistically significant strong correlation with CT indwelling time (days) $(\mathrm{P}<0.0001)$ and CT total effusion $(\mathrm{mL})(\mathrm{P}<0.0001)$.

The linear regression model was fitted so as to estimate the contribution of explicative variables in describing the surgical OR time, global OR time and pLOS. As shown in Table 3, A, the results from the linear regression model indicated that patients ID, BMI and the presence of adhesions contributed to explain the values of surgical OR time, and that explicative variables statistically significant contributed to estimate the time. In particular, considering the progressive number of admitted patients, the required time for surgical operation significantly decreased by $0.2 \min (\mathrm{P}=0.003)$; on the other hand, each point of increase in BMI statistically significantly lengthened the surgical OR 
Table 2 Descriptive statistics of peri-operative anesthesia and analgesia management in NIVATS uniportal lung biopsy for undetermined ILD

\begin{tabular}{|c|c|}
\hline Variable & $\begin{array}{l}\text { ILD patients } \\
(\mathrm{n}=99)\end{array}$ \\
\hline Conversion to general anesthesia, $\mathrm{n}[\%]$ & $0[0]$ \\
\hline $\begin{array}{l}\text { Intra-operative peripheral oxygenation (\%), } \\
\text { mean (SD) }\end{array}$ & $97.2(2.2)$ \\
\hline Intra-operative $\mathrm{O}_{2}$ supply (L/min), mean (SD) & $3.6(1.5)$ \\
\hline \multicolumn{2}{|l|}{ Intra-operative anesthesia medications, $\mathrm{n}$ [\%] } \\
\hline Midazolam & 9 [9] \\
\hline Fentanyl & 2 [2] \\
\hline Remifentanil & $1[1]$ \\
\hline Propofol & $1[1]$ \\
\hline Midazolam + fentanyl & 9 [9] \\
\hline Midazolam + remifentanil & 15 [15] \\
\hline Remifentanil + propofol & 3 [3] \\
\hline Midazolam + fentanyl + remifentanil & $30[30]$ \\
\hline Midazolam + fentanyl + propofol & $1[1]$ \\
\hline Midazolam + remifentanil + propofol & $13[13]$ \\
\hline Midazolam + fentanyl + remifentanil + propofol & 15 [15] \\
\hline \multicolumn{2}{|l|}{ Locoregional analgesia, $\mathrm{n}[\%]$} \\
\hline Paravertebral catheter & $4[4]$ \\
\hline Intercostal nerve block & $38[38]$ \\
\hline None & $57[58]$ \\
\hline Post-operative analgesia supply, n [\%] & $57[57]$ \\
\hline Paracetamol supply & $42[74]$ \\
\hline Nonsteroidal anti-inflammatory drug supply & 52 [91] \\
\hline Mean Numeric Pain Rate Scale (score), mean (SD) & $1.13(0.82)$ \\
\hline
\end{tabular}

Data is represented as mean (SD) or number [\%], based on variable distribution. NIVATS, non-intubated video-assisted thoracic surgery; ILD, interstitial lung disease.

time of $1.27 \mathrm{~min}(\mathrm{P}=0.003)$. Furthermore, the increase of a single point in $\mathrm{FEV} 1 \%$ resulted in a decrease of surgical OR time of $0.20 \mathrm{~min}(\mathrm{P}=0.018)$.

A statistically significant increase of $12 \mathrm{~min}$ in surgical OR time was observed in patients with adhesions compared with those without $(\mathrm{P}=0.010)$. No statistically significant effect was found when analyzing possible correlation between surgical OR time and the administration of intra-operative anesthesia drugs (midazolam, fentanyl, remifentanil, propofol when administered either alone, or in combination).

The second linear regression model was fitted in order to estimate the contribution of explicative variables in describing the global OR time. As reported in Table 3, B, the BMI influenced the global OR time with a statistically significant delay of approximately $1.7 \mathrm{~min}$ per each point of increase in BMI $(\mathrm{P}=0.003)$. Conversely, the global OR time showed a reduction of $0.4 \mathrm{~min}$ per each subsequently admitted patient $(\mathrm{P}<0.0001)$. In addition, the administration of Remifentanil statistically significantly reduced the global OR time by about $30 \mathrm{~min}(\mathrm{P}<0.0001)$ if used alone, according to the management of anesthesia.

The third linear regression model estimated the pLOS based on the effects of ID, BMI, CHs and CT indwelling time. As showed in Table 3, C, the reduction of post-operative in-hospital length of stay was statistically significantly associated with an increment of progressive number of patient $(\mathrm{P}=0.005)$. Contrariwise, an approximate increment of 1 day in pLOS was observed when increasing the CT indwelling time $(\mathrm{P}<0.0001)$ and approximately one additional day in pLOS is associated with post-operative complications $(\mathrm{P}<0.0001)$. No statistically significant effects have been found by the model when considering possible influences of $\mathrm{CHs}$ and $\mathrm{BMI}$ on the pLOS.

\section{Locoregional anesthesia versus local anesthesia alone}

Considering the diverse management in locoregional analgesia as reported in Table 2, a total of 38 (38\%) patients underwent IB, 4 (4\%) were treated with paravertebral catheter (PVC) positioning and 57 (58\%) did not receive locoregional analgesia. Since only four patients were subjected to PVC, these were excluded from this secondary analysis (Table 4) in order to ensure an unbiased evaluation. Group A was made up of patients that underwent IB, while the rest of the patients were included in group B. Surgical OR time, global OR time, pLOS, NPRS and post-operative rescue analgesia were evaluated by group. A statistically significant reduction $(\mathrm{P}=0.021)$ of global $\mathrm{OR}$ time (mean $=59 \mathrm{~min}, \mathrm{SD}=18 \mathrm{~min}$ ) was detected in patients subjected to IB (group A), compared to group B (mean =79 $\mathrm{min}, \mathrm{SD}=37$ min), as well as surgical OR time (group A: mean $=38 \mathrm{~min}$, $\mathrm{SD}=12 \mathrm{~min}$, group $\mathrm{B}$ : mean $=46 \mathrm{~min}, \mathrm{SD}=24 \mathrm{~min}$ ), but the difference is borderline $(\mathrm{P}=0.051)$ as displayed in Figure 3. No statistically significant differences were detected in NPRS between groups $(\mathrm{P}=0.087)$, neither in pLOS 
Table 3 Results of linear regression model aimed at estimating the distribution of the different surgical times and in-hospital stay

\begin{tabular}{|c|c|c|c|}
\hline Variable & Coeff. & $95 \% \mathrm{Cl}$ & $P$ value \\
\hline \multicolumn{4}{|l|}{ A) Surgical OR time (min) } \\
\hline ID (patients enrolment progression) & -0.204 & $-0.337,-0.071$ & 0.003 \\
\hline BMI & 1.270 & $0.446,2.094$ & 0.003 \\
\hline Adhesions (yes) & 12.173 & $3.082,21.263$ & 0.009 \\
\hline \multicolumn{4}{|l|}{ B) Global OR time (min) } \\
\hline ID (patients enrolment progression) & -0.435 & $-0.659,-0.212$ & $<0.0001$ \\
\hline BMI & 1.689 & $0.582,2.797$ & 0.003 \\
\hline Remifentanil (alone) & -29.569 & $-44.946,-14.192$ & $<0.0001$ \\
\hline BMI & 0.014 & $0.008,0.036$ & 0.214 \\
\hline Chest tube indwelling time (days) & 0.885 & $0.741,1.030$ & $<0.0001$ \\
\hline Clavien-Dindo complication (yes) & 0.868 & $0.492,1.245$ & $<0.0001$ \\
\hline Charlson comorbidity (score) & -0.040 & $-0.092,-0.010$ & 0.115 \\
\hline
\end{tabular}

A) results of the linear regression model for surgical OR time as the outcome; B) results of linear regression model for global OR time as the outcome; C) results of linear regression model for pLOS as the outcome. Data is presented as regression coefficient ( $\beta$ ), $95 \%$ confidence interval $(95 \% \mathrm{Cl})$ and $\mathrm{P}$ value. OR, operating Room; pLOS, post-operative length of stay; BMI, body mass index; FEV1\%, forced expiratory volume in the 1 st second percentage.

Table 4 Post-operative outcomes between patients treated with intercostal blockage (group A) and local anesthesia only (group B)

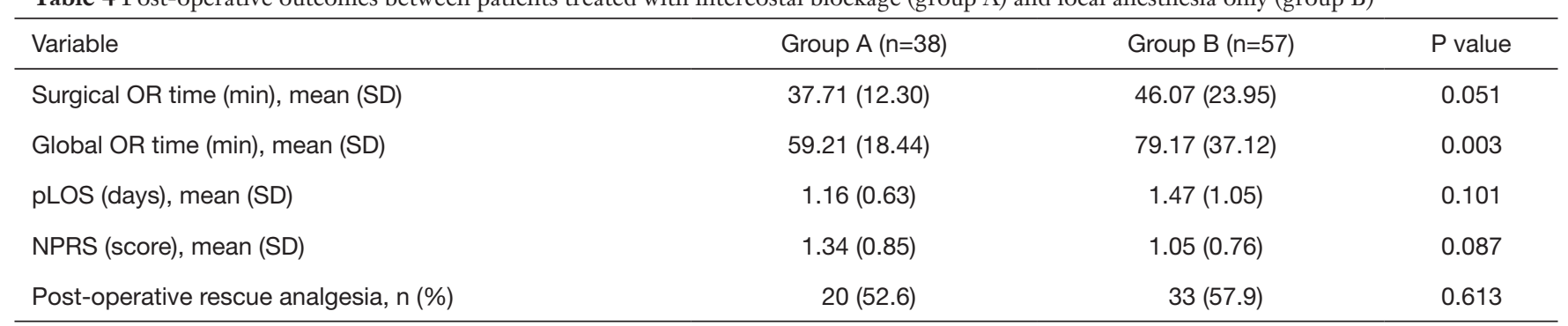

OR, operating room; pLOS, post-operative length of stay; NPRS, Numeric Pain Rate Scale.

$(\mathrm{P}=0.101)$ nor in the demand for post-operative rescue analgesia $(\mathrm{P}=0.169)$.

\section{Outcome results with different CT sizes}

No statistically significant differences were detected in post-operative outcomes such as pLOS, post-operative complications or NPRS patient feedback when considering the different caliber of CT that were placed intraoperatively (Table 5).

\section{Discussion}

In our series, which according to a brief literature review seems to be the largest reported by a single Institute, the data regarding diagnostic yield of NIVATS lung biopsy appears to be comparable to that achieved by VATS lung biopsy in GA. Moreover, secondary outcomes as early mortality, recorded a lower incidence than that described in other large series of intubated VATS lung biopsy procedures. 

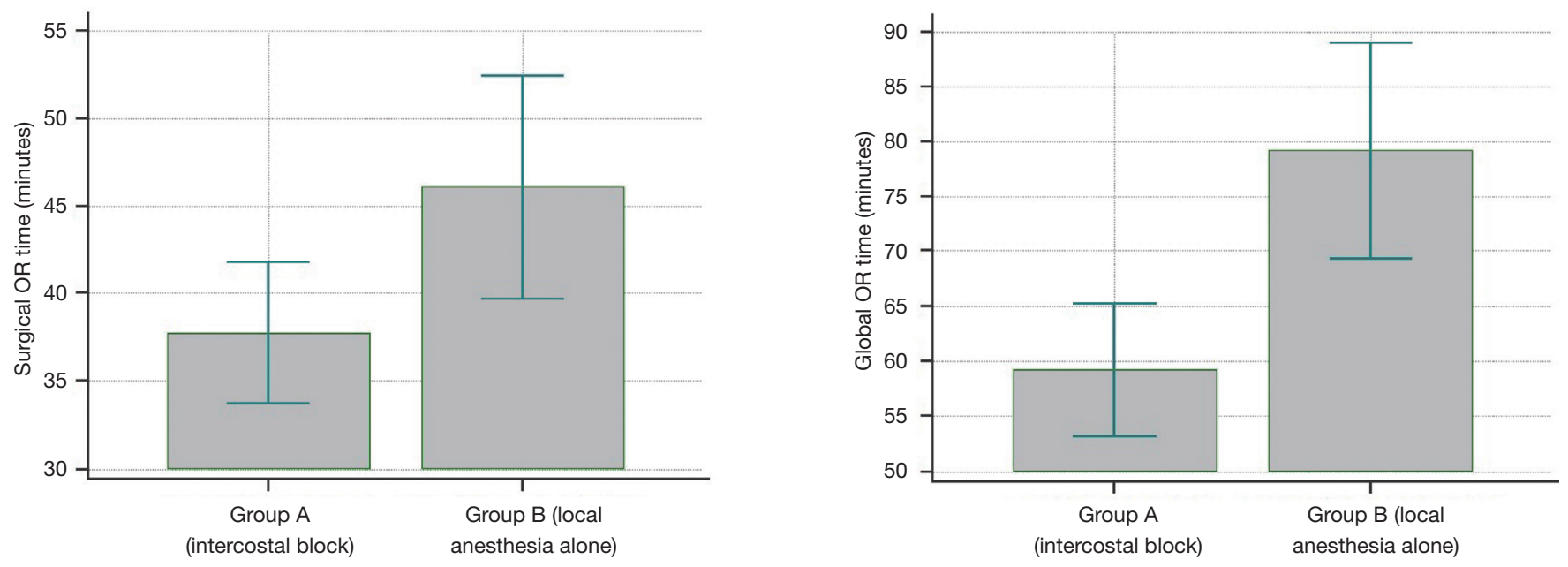

Figure 3 Global operating room (OR) length of stay and surgical operating time comparison between patients treated with or without intercostal nerve blockage anesthesia.

Table 5 Post-operative outcomes between patients drained with small chest tubes (19 or 21 French) and large chest tubes (28 French)

\begin{tabular}{|c|c|c|c|}
\hline Variable & Small CT $(n=32)$ & Large CT $(n=67)$ & $P$ value \\
\hline NPRS (score), mean (SD) & $1.06(0.71)$ & $1.17(0.87)$ & 0.511 \\
\hline Post-operative complications, n (\%) & $4(12.5)$ & $5(7.5)$ & 0.415 \\
\hline
\end{tabular}

pLOS, post-operative length of stay; NPRS, Numeric Pain Rate Scale.

Considering the most relevant intra-operative results, the surgical operative time seems to be influenced by the learning curve of the surgical team, underlining the importance of sound teamwork between surgeons and anesthesiologists. Despite pleural adhesions suggesting the need to delay surgery as well as higher values of BMI and poorer PFT no intra-operative complications occurred.

The management of anesthesia with a standardized algorithm of TIVA, using continuous infusion of propofol associated with remifentanil and fentanyl to maintain the sedation, appears to be feasible and reproducible. Moreover, it seems to be safe as evidenced by the lack of necessity for intubation or conversion to GA.

In our opinion, the absence of statistically significant differences between patients who underwent IB and those who did not, is remarkably interesting in terms of post-operative in-hospital NPRS feedback or the supply of analgesia medication. The SLB procedure in nonintubated patients was confirmed to be less invasive with a small impact on the perception of pain by the patient. Otherwise, IB should facilitate the surgical procedure with a better management of patient discomfort due to surgical manoeuvres, as well as a faster OR discharge as a result of a swift recovery from sedation.

The average pLOS in our series was comparable to the results of other studies where the non-intubated lung biopsy technique had been applied for ILD patients (15-19). Among several baseline characteristic analysed, only age of patients, BMI and PFTs influenced the duration of postoperative in-hospital stay. Obviously, even minor postoperative complications and the CT indwelling time directly delayed the discharge of the patient, while the different size of CT positioned did not influence the postoperative outcomes that were explored.

The early mortality rate did not appear to be different from other small series of patients underwent NIVATS lung biopsy, as reported in the literature (17). Considering this aspect, preliminary data suggests even better outcomes when compared to VATS lung biopsy in intubated ILD patients (1).

Patients with ILD, are usually associated with critical clinical conditions dictated by impaired respiratory 



Figure 4 Histopathologic hallmark of usual interstitial pneumonia (UIP). (A) HE 10×. Architectural remodelling as a result of patchy dense fibrosis in peripheral area inside the lobule and the perivenular area. Microscopic honeycombing cysts noted; (B) HE 40×. The fibroblastic focus consists of fibroblasts and myofibroblasts and are covered by type II pneumocytes or cuboidal metaplastic epithelia. Histopathology Department, “A. Businco" Oncology Hospital, Cagliari (IT).

function (1). A correct diagnosis is considered to be a cornerstone in the therapeutic approach to ILD, with regard to suspected IPF. Unfortunately, the high diagnostic sensitivity achieved with HRCT remains a peculiarity of few referral centres with a high volume of ILD/IPF casings and consequent high level of expertise. Due to the discrepancies in radio-diagnostic skills, unidentified ILD cases require a mandatory lung biopsy in order to achieve a diagnostic certainty (20). Moreover, it is commonly though that a biopsy late in the course of the disease is less effective and more hazardous (21). A multidisciplinary, dynamic approach, with the input of clinicians, radiologists, and pathologists has proven to significantly improve diagnostic accuracy and is strongly recommended (22). In that context, a precise histopathological characterization by SLB can help in orientating therapy and can reliably predict prognosis.
However, prolonged surgical pulmonary manipulation, hyperoxia, atelectrauma and barotrauma derived by selective lung mechanical ventilation, should represent some of the possible physio-pathological triggers related to post-surgical acute lung disease injury and acute respiratory distress syndrome, that carry a high mortality rate in this set of patients (23-25). Even with VATS lung biopsy procedures, it is important to be aware of the possibility of post-surgical acute exacerbation of interstitial pneumonia, even after an apparently uneventful immediate post-operative course, with a reported incidence from $2.1 \%$ to $7 \%$ in the most representative series. These events are associated with a mortality range between $80 \%$ and $90 \%$ in IPF patients (26).

Despite recent enthusiasm for new techniques such as transbronchial lung cryobiopsy (TBLC), the comparison with VATS lung biopsy as the current standard for diagnosis in ILD patients, represents a topical debate (27). The relatively low risk of operative death and post-operative respiratory failure connected to elective thoracoscopic biopsy are contrary to the current lack of standardization of technique and unclear outcomes and advantages related to TBLC $(28,29)$. Recent studies and meta-analysis showed a pooled estimated diagnostic yield typically from $71 \%$ to $83 \%$ for TBLC (30), still not comparable with that achieved by SLB. Furthermore, study to assess diagnostic concordance between TBLC and SLB sequentially performed in the same patients, did not show acceptable results (31). A considerable percentage of patients with uncertain ILD diagnosis that underwent non-surgical biopsy procedures, must be referred to a surgeon in order to minimize structural artifacts of the tissue in lung specimens for a better histopathological examination (Figure $4 A, B$ ).

Nowadays, video-assisted thoracic surgery in nonintubated patients is being increasingly employed when performing lung procedures, including lobectomy, wedge resection, management of the pneumothorax, and lung volume reduction surgery (32). The most attractive rationale is that avoiding GA reduces its related side effects, possibly resulting in a shorter hospital stay and lower operative risk, particularly in patients with compromised respiratory function $(33,34)$. From the initial series of Pompeo et al. (15), other groups investigated the feasibility and efficacy of minimally invasive SLB in tubeless ILD patients with or without loco regional anesthesia, resulting in low morbidity, excellent diagnostic yield, shorter hospital stay and low costs (16-19,35). Thoracoscopic SLB procedure with local anesthesia and sedation allows a better patient's physical 
state monitoring and communication $(17,36)$. Other advantages include the ability to cough throughout the procedure, promoting better ventilation, and is associated with fewer respiratory complications and faster recovery time (37). Therefore, local anesthesia appears to be less traumatic for the immune system, allowing for a shorter recovery time (38). In the present study, we agree with the idea that an inexperienced surgical team with poor cooperative skills may be the only real difficulties in performing NIVATS lung biopsy. The cooperation and coordination between surgeons and anesthesiologists is fundamental to avoid problems and mistakes, which can seriously put at risk the safety of patients and the postoperative outcomes (39).

Unfortunately, little evidence is available in the literature for an accurate evaluation of NIVATS lung biopsy feasibility and the benefits in ILD/IPF patients, as it is based on small series and retrospective studies. The data collected from other large series of NIVATS procedures including lung biopsies do not clarify post-operative outcomes $(40,41)$, although the results seem to encourage this kind of lessinvasive approach.

\section{Limitations}

The results of our study include a retrospective analysis of the data that was collected at our centre but lack a control group made up of lung biopsies in intubated VATS patients. Furthermore, the study was carried out in one single Institute, where the succession of patients subjected to the NIVATS lung biopsies may have influenced the team's ever-growing expertise, as demonstrated by results related to progressive patient ID. On the other, the existence of a standardized surgical and peri-operative management protocol, could ensure a homogeneous criterion in data collection and outcome analysis.

\section{Conclusions}

The study showed that the NIVATS lung biopsy technique is easily executed with a low complication rate, and reduces post-operative patient discomfort and hospital stay even in patients who did not receive IB anesthesia. According to current indications for ILD diagnosis, this technique should be considered as a valid alternative to the standard VATS approach. Larger perspective studies with adequate control groups are needed to assess the long-term effects and diagnostic rate of this less-invasive surgical strategy.

\section{Acknowledgments}

Funding: None.

\section{Footnote}

Reporting Checklist: The authors have completed the STROBE reporting checklist. Available at http://dx.doi. org/10.21037/jtd-20-1551

Data Sharing Statement: Available at http://dx.doi. org/10.21037/jtd-20-1551

Conflicts of Interest: All authors have completed the ICMJE uniform disclosure form (available at http://dx.doi. org/10.21037/jtd-20-1551). The authors have no conflicts of interest to declare.

Ethical Statement: The authors are accountable for all aspects of the work in ensuring that questions related to the accuracy or integrity of any part of the work are appropriately investigated and resolved. The study was approved by the Institutional Review Board (Reference Ethics Committee No. PG/2017/16770) and conducted in accordance with the Declaration of Helsinki (as revised in 2013). Informed written consent was obtained from all the patients.

Open Access Statement: This is an Open Access article distributed in accordance with the Creative Commons Attribution-NonCommercial-NoDerivs 4.0 International License (CC BY-NC-ND 4.0), which permits the noncommercial replication and distribution of the article with the strict proviso that no changes or edits are made and the original work is properly cited (including links to both the formal publication through the relevant DOI and the license). See: https://creativecommons.org/licenses/by-nc-nd/4.0/.

\section{References}

1. Raghu G, Remy-Jardin M, Myers JL, et al. Diagnosis of Idiopathic Pulmonary Fibrosis. An Official ATS/ERS/JRS/ ALAT Clinical Practice Guideline. Am J Respir Crit Care Med 2018;198:e44-68.

2. Flaherty KR, King TE Jr, Raghu G, et al. Idiopathic interstitial pneumonia: what is the effect of a multidisciplinary approach to diagnosis? Am J Respir Crit Care Med 2004;170:904-10.

3. Jo HE, Glaspole IN, Levin KC, et al. Clinical impact 
of the interstitial lung disease multidisciplinary service. Respirology 2016;21:1438-44.

4. Walsh SL, Wells AU, Desai SR, et al. Multicentre evaluation of multidisciplinary team meeting agreement on diagnosis in diffuse parenchymal lung disease: a casecohort study. Lancet Respir Med 2016;4:557-65.

5. Cottin V. Lung biopsy in interstitial lung disease: balancing the risk of surgery and diagnostic uncertainty. Eur Respir J 2016;48:1274-7.

6. Nason KS. Considering what we know, diagnostic yield and postoperative mortality associated with surgical lung biopsy for evaluation of interstitial lung diseases are good ... we think .... J Thorac Cardiovasc Surg 2015;149:1402-3.

7. Hutchinson JP, McKeever TM, Fogarty AW, et al. Surgical lung biopsy for the diagnosis of interstitial lung disease in England: 1997-2008. Eur Respir J 2016;48:1453-61.

8. Lettieri CJ, Veerappan GR, Helman DL, et al. Outcomes and safety of surgical lung biopsy for interstitial lung disease. Chest 2005;127:1600-5.

9. Endo S, Bando M. Invited commentary: Videothoracoscopy-assisted surgical lung biopsy for interstitial lung diseases. Gen Thorac Cardiovasc Surg 2014;62:331-3.

10. Raj R, Brown KK. Mortality Related to Surgical Lung Biopsy in Patients with Interstitial Lung Disease. The devil is the Denominator. Am J Respir Crit Care Med 2016;193:1082-4.

11. Yang SM, Wang ML, Hung MH, et al. Tubeless Uniportal Thoracoscopic Wedge Resection for Peripheral Lung Nodules. Ann Thorac Surg 2017;103:462-8.

12. Lirio F, Galvez C, Boloufer S, et al. Tubeless major pulmonary resections. J Thorac Dis 2018;10:S2664-70.

13. Ramsay MA, Savage TM, Simpson BR, et al. Controlled sedation with alphaxone-alphadone. Br Med J 1974;2:656-9.

14. Dindo D, De Martines N, Clavien PA. Classification of surgical complications: a new proposal with evaluation in a cohort of 6336 patients and results of a survey. Ann Surg 2004;240:205-13.

15. Pompeo E, Rogliani P, Cristino B, et al. Awake thoracoscopic biopsy of interstitial lung disease. Ann Thorac Surg 2013;95:445-52.

16. Hajjar WM, Al-Nassar SA, Al-Sugair GS, et al. Evaluation of safety and efficacy of regional anaesthesia compared with general anaesthesia in thoracoscopic lung biopsy procedure on patient with idiopathic pulmonary fibrosis. Saudi J Anaesth 2018;12:46-51.
17. Pompeo E, Rogliani P, Atinkaya C, et al. Nonintubated surgical biopsy of undetermined interstitial lung disease: a multicentre outcome analysis. Interact Cardiovasc Thorac Surg 2019;28:744-50.

18. Jeon CS, Yoon DW, Moon SM, et al. Non-intubated video-assisted thoracoscopic lung biopsy for interstitial lung disease: a single-center experience. J Thorac Dis 2018;10:3262-8.

19. Peng G, Liu M, Luo Q, et al. Spontaneous ventilation anaesthesia combined with uniportal and tubeless thoracoscopic lung biopsy in selected patients with interstitial lung diseases. J Thorac Dis 2017;9:4494-501.

20. Vaszar LT, Larsen BT, Swanson KL, et al. Diagnostic utility of surgical lung biopsies in elderly patients with indeterminate interstitial lung disease. Respirology 2018;23:507-11.

21. Lieberman S, Gleason JB, Ilyas MIM, et al. Assessing the Safety and Clinical Impact of Thoracoscopic Lung Biopsy in Patients with Interstitial Lung Disease. J Clin Diagn Res 2017;11:OC57-9.

22. Lynch DA, Sverzellati N, Travis WD, et al. Diagnostic criteria for idiopathic pulmonary fibrosis: a Fleischner Society White Paper. Lancet Respir Med 2018;6:138-53.

23. Ghatol A, Ruhl P, Danoff S. Exacerbations in Idiopathic Pulmonary Fibrosis Triggered by Pulmonary and Nonpulmonary Surgery: A Case Series and Comprehensive Review of the Literature. Lung 2012;190:373-80.

24. Dreyfuss D, Saumon G. Role of tidal volume, FRC, and end-inspiratory volume in the development of pulmonary edema following mechanical ventilation. Am Rev Respir Dis $1993 ; 148: 1194-203$.

25. Amato MB, Barbas CS, Medeiros DM, et al. Effect of a protective-ventilation strategy on mortality in the acute respiratory distress syndrome. N Engl J Med 1998;338:347-54.

26. Han Q, Luo Q, Xie JX, et al. Diagnostic yield and postoperative mortality associated with surgical lung biopsy for evaluation of interstitial lung diseases: A systematic review and meta-analysis. J Thorac Cardiovasc Surg 2015;149:1394-401.e1.

27. Ravaglia C, Bonifazi M, Wells AU, et al. Safety and Diagnostic Yield of Transbronchial Lung Cryobiopsy in Diffuse Parenchymal Lung Diseases: A Comparative Study versus Video-Assisted Thoracoscopic Lung Biopsy and a Systematic Review of the Literature. Respiration 2016;91:215-27.

28. Poletti V, Hetzel J. Transbronchial Cryobiopsy in Diffuse Parenchymal Lung Disease: Need for Procedural 
Standardization. Respiration 2015;90:275-8.

29. Lentz RJ, Taylor TM, Kropski JA, et al. Utility of Flexible Bronchoscopic Cryobiopsy for Diagnosis of Diffuse Parenchymal Lung Diseases. J Bronchology Interv Pulmonol 2018;25:88-96.

30. Johannson KA, Marcoux VS, Ronksley PE, et al. Diagnostic Yield and Complications of Transbronchial Lung Cryobiopsy for Interstitial Lung Disease. A Systematic Review and Metaanalysis. Ann Am Thorac Soc 2016;13:1828-38.

31. Romagnoli M, Colby TV, Berthet JP, et al. Poor Concordance between Sequential Transbronchial Lung Cryobiopsy and Surgical Lung Biopsy in the Diagnosis of Diffuse Interstitial Lung Diseases. Am J Respir Crit Care Med 2019;199:1249-56.

32. Bertolaccini L, Zaccagna G, Divisi D, et al. Awake nonintubated thoracic surgery: an attempt of systematic review and meta-analysis. Video-assist Thorac Surg 2017;2:59

33. Lan L, Cen Y, Zhang C, et al. A Propensity ScoreMatched Analysis for Non-Intubated Thoracic Surgery. Med Sci Monit 2018;24:8081-7.

34. Ali JM, Volpi S, Kaul P, et al. Does the 'non-intubated' anaesthetic technique offer any advantage for patients undergoing pulmonary lobectomy? Interact Cardiovasc

Cite this article as: Cherchi R, Grimaldi G, Pinna-Susnik M, Riva L, Sarais S, Santoru M, Perra R, Allieri R, Porcu GS, Nemolato S, Mameli A, Loi F, Ferrari PA. Retrospective outcomes analysis of 99 consecutive uniportal awake lung biopsies: a real standard of care? J Thorac Dis 2020;12(9):47174730. doi: $10.21037 /$ jtd-20-1551
Thorac Surg 2019;28:555-8.

35. Ambrogi V, Mineo TC. VATS biopsy for undetermined interstitial lung disease under non-general anesthesia: comparison between uniportal approach under intercostal block vs. three-ports in epidural anesthesia. J Thorac Dis 2014;6:888-95.

36. Wang B, Ge S. Nonintubated anesthesia for thoracic surgery. J Thorac Dis 2014;6:1868-74.

37. Yang JT, Hung MH, Chen JS, et al. Anesthetic consideration for nonintubated VATS. J Thorac Dis 2014;6:10-3.

38. Mineo TC, Ambrogi V. Immune effects after uniportal nonintubated video-thoracoscopic operations. Video-assist Thorac Surg 2018;3:4.

39. Tacconi F, Mineo TC, Ambrogi V. Team Training for Nonintubated Thoracic Surgery. Thorac Surg Clin 2020;30:111-20.

40. Mineo TC, Tamburrini A, Perroni G, et al. 1000 cases of tubeless video-assisted thoracic surgery at the Rome Tor Vergata University. Future Oncol 2016;12:13-8.

41. Katlic MR, Facktor MA. Video-assisted thoracic surgery utilizing local anesthesia and sedation: 384 consecutive cases. Ann Thorac Surg 2010;90:240-5. 
Table S1 Pearson's correlation coefficients between independent variables considered in the study

\begin{tabular}{|c|c|c|c|c|c|c|c|c|c|c|c|}
\hline Variable & ID & $\begin{array}{l}\text { Global OR time } \\
\text { (min) }\end{array}$ & $\begin{array}{l}\text { Surgical OR } \\
\text { time (min) }\end{array}$ & pLOS (days) & BMI & Age (years) & FEV1\% & FVC\% & $\begin{array}{c}\text { Charlson } \\
\text { comorbidity (score) }\end{array}$ & $\begin{array}{l}\text { Chest tube total } \\
\text { effusion }(\mathrm{mL})\end{array}$ & $\begin{array}{c}\text { Chest tube } \\
\text { indwelling time (days) }\end{array}$ \\
\hline ID & - & - & - & - & - & - & - & - & - & - & - \\
\hline Global OR time (min) & $\begin{array}{c}-0.5864 \\
(P<0.0001)^{\star}\end{array}$ & - & - & - & - & - & - & - & - & - & - \\
\hline Surgical OR time (min) & $\begin{array}{c}-0.3033 \\
(P=0.0023)^{\star}\end{array}$ & $\begin{array}{c}0.5834 \\
(P<0.0001)^{\star}\end{array}$ & - & - & - & - & - & - & - & - & - \\
\hline pLOS (days) & $\begin{array}{c}0.0459 \\
(P=0.6499)\end{array}$ & $\begin{array}{c}0.0835 \\
(P=0.4115)\end{array}$ & $\begin{array}{c}0.0254 \\
(P=0.8028)\end{array}$ & - & - & - & - & - & - & - & - \\
\hline BMI & $\begin{array}{c}-0.0401 \\
(P=0.7985)\end{array}$ & $\begin{array}{c}0.2156 \\
(P=0.0321)^{\star}\end{array}$ & $\begin{array}{c}0.2963 \\
(\mathrm{P}=0.0029)^{*}\end{array}$ & - & - & - & - & - & - & - & - \\
\hline Age (years) & $\begin{array}{c}0.0177 \\
(P=0.8614)\end{array}$ & $\begin{array}{c}0.0191 \\
(P=0.8508)\end{array}$ & $\begin{array}{c}0.1221 \\
(P=0.2286)\end{array}$ & $\begin{array}{c}-0.2834 \\
(P=0.0043)^{\star}\end{array}$ & $\begin{array}{c}0.1937 \\
(P=0.0534)\end{array}$ & - & - & - & - & - & - \\
\hline FEV1\% & $\begin{array}{c}-0.0542 \\
(P=0.6160)\end{array}$ & $\begin{array}{c}-0.1633 \\
(P=0.1285)\end{array}$ & $\begin{array}{c}-0.2237 \\
(P=0.0372)^{*}\end{array}$ & $\begin{array}{c}-0.2282 \\
(P=0.0325)^{\star}\end{array}$ & $\begin{array}{c}-0.0041 \\
(P=0.9698)\end{array}$ & $\begin{array}{c}0.2392 \\
(P=0.0248)\end{array}$ & - & - & - & - & - \\
\hline FVC\% & $\begin{array}{c}-0.0575 \\
(\mathrm{P}=0.5948)\end{array}$ & $\begin{array}{c}-0.1151 \\
(P=0.2856)\end{array}$ & $\begin{array}{c}-0.2161 \\
(P=0.0444)^{*}\end{array}$ & $\begin{array}{c}-0.2487 \\
(P=0.0195)^{\star}\end{array}$ & $\begin{array}{c}-0.0532 \\
(P=0.6624)\end{array}$ & $\begin{array}{c}0.0578 \\
(P=0.5930)\end{array}$ & $\begin{array}{c}0.8953 \\
(P<0.0001)^{\star}\end{array}$ & - & - & - & - \\
\hline $\begin{array}{l}\text { Charlson comorbidity } \\
\text { (score) }\end{array}$ & $\begin{array}{c}0.0154 \\
(P=0.8790)\end{array}$ & $\begin{array}{c}0.0035 \\
(P=0.9722)\end{array}$ & $\begin{array}{c}0.0437 \\
(P=0.6676)\end{array}$ & $\begin{array}{c}-0.1887 \\
(P=0.0601)\end{array}$ & $\begin{array}{c}0.0028 \\
(P=0.9797)\end{array}$ & $\begin{array}{c}0.5682 \\
(P<0.0001)^{\star}\end{array}$ & $\begin{array}{c}-0.0150 \\
(P=0.8900)\end{array}$ & $\begin{array}{c}-0.0833 \\
(P=0.4403)\end{array}$ & - & - & - \\
\hline $\begin{array}{l}\text { Chest tube total } \\
\text { effusion }(\mathrm{mL})\end{array}$ & $\begin{array}{c}0.0120 \\
(P=0.9058)\end{array}$ & $\begin{array}{c}0.1678 \\
(\mathrm{P}=0.0915)\end{array}$ & $\begin{array}{c}-0.0139 \\
(P=0.8916)\end{array}$ & $\begin{array}{c}0.6147 \\
(P<0.0001)^{\star}\end{array}$ & $\begin{array}{c}-0.2669 \\
(P=0.0073)^{\star}\end{array}$ & $\begin{array}{c}-0.1024 \\
(P=0.3108)\end{array}$ & $\begin{array}{c}-0.2192 \\
(P=0.0402)^{*}\end{array}$ & $\begin{array}{c}-0.2289 \\
(P=0.0319)^{\star}\end{array}$ & $\begin{array}{c}-0.0730 \\
(P=0.4705)\end{array}$ & - & - \\
\hline $\begin{array}{l}\text { Chest tube indwelling } \\
\text { time (days) }\end{array}$ & $\begin{array}{c}0.2191 \\
(P=0.0285)\end{array}$ & $\begin{array}{c}-0.0308 \\
(P=0.7610)\end{array}$ & $\begin{array}{c}-0.1181 \\
(P=0.0622)\end{array}$ & $\begin{array}{c}0.8064 \\
(P<0.0001)^{\star}\end{array}$ & $\begin{array}{c}-0.1369 \\
(P=0.1744)\end{array}$ & $\begin{array}{c}-0.2381 \\
(P=0.0171)^{\star}\end{array}$ & $\begin{array}{c}-0.2203 \\
(P=0.0392)^{\star}\end{array}$ & $\begin{array}{c}-0.2205 \\
(P=0.0390)^{*}\end{array}$ & $\begin{array}{c}-0.1313 \\
(\mathrm{P}=0.1930)\end{array}$ & $\begin{array}{c}0.6616 \\
(P<0.0001)^{\star}\end{array}$ & - \\
\hline
\end{tabular}

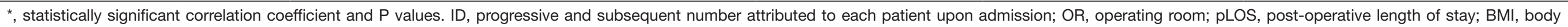
mass index; FEV1\%, forced expiratory volume in the 1st second percentage; FVC\%, forced volume capacity percentage. 